\title{
Role of bevacizumab therapy in the management of glioblastoma
}

\author{
This article was published in the following Dove Press journal: \\ Cancer Management and Research \\ 21 April 2010 \\ Number of times this article has been viewed
}

\section{Scott J Peak \\ Victor A Levin}

Neuro-Oncology Program, Department of Neurosurgery and Neuroscience, Kaiser Permanente, Redwood City, CA, USA
Correspondence: Victor A Levin Department of Neurosciences, Kaiser Permanente, I I 50 Veterans Blvd., Redwood City, CA 94025, USA

Tel + I 650-299-3665

$\mathrm{Fax}+\mathrm{I}$ 650-299-2677

Email vlevin49@comcast.net
Abstract: Glioblastoma is one of the most common primary brain tumors and one of the most difficult to treat. In population-based studies only $30 \%$ of patients will survive 1 year and in the most efficacious surgery, irradiation, and chemotherapy clinical trials approximately $20 \%$ will live 2 years. Bevacizumab is a recombinant, antivascular epidermal growth factor receptor (VEGF) monoclonal antibody with 6 VEGF-binding residues that binds to VEGF, preventing VEGF from binding to its target, VEGFR-1 and VEGFR-2, on endothelial cells. Through its binding to VEGF ligands bevacizumab reduces tumor angiogenesis and vasogenic brain edema; the consequences are that bevacizumab reduces the rate of glioblastoma tumor growth and its associated tumoral edema, thereby improving quality of life and survival for patients suffering from cerebral glioblastoma. In this review, we will summarize the studies that led to the use of bevacizumab in glioblastoma and the potential side-effects and complications that can be associated with its use and, finally, new opportunities for drug combinations with bevacizumab.

Keywords: chemotherapy, VEGF, edema, central nervous system

\section{Introduction}

Glioblastoma multiforme $(\mathrm{GBM})$ is a primary brain tumor arising from cells of astrocytic lineage. GBM is one of the most common and aggressive malignancies of the central nervous system (CNS). Despite its aggressiveness, there are molecular differences among all glioblastomas that result in a range of responsiveness to treatment. Primary glioblastomas arise without a preceding low grade glioma, often show amplification of epidermal growth factor receptor (EGFR) and murine double minute 2 (MDM2), along with inactivation of phosphatase and tensin homolog (PTEN) and p16 tumor suppressor genes. Secondary glioblastomas often have loss of p53 and pRB, as well as an increase in CDK4/6 expression. In 2005, Hegi and colleagues were able to show that outcome for patients with newly diagnosed glioblastomas depended on the methylation status of O6-methylguanine DNA methyltransferase (MGMT), with median survival increasing to 21.7 months in those with methylation of MGMT treated with both external beam irradiation and temozolomide. ${ }^{1}$ As a result, molecular profiling of glioblastoma tissue collected at surgery is becoming important to patient treatment considerations and care.

The incidence rate of GBM is 3.1 per 100,000 person-years, ${ }^{2}$ overall incidence of gliomas is greater in males (7.2 per 100,000 person-years) compared to females (5.0 per 100,000 person-years) and increases with age to $14 / 100,000$ over age 65 . The incidence rate is also greater amongst Caucasians compared to other ethnicities, and $18.5 \%$ of all brain tumors are GBM. ${ }^{2}$ 
The median age at diagnosis of GBM is 64 years, and GBM is the second most common primary brain tumor behind only meningioma in patients at least 45 years of age. ${ }^{2}$ Based on general tumor registry data, approximately $29.6 \%$ of patients with GBM will survival 1 year, whereas only $3.4 \%$ will survive 5 years with treatment. ${ }^{2}$

Studies evaluating risk factors for brain tumors have thus far shown previous exposure to ionizing radiation as a statistically significant risk factor, but no conclusive evidence yet supports electromagnetic fields, cell phones, neurocarcinogens or metals as increasing risk of brain tumor development. ${ }^{3}$ There are specific genetic disorders that are associated with an increased risk for developing malignancies, including brain tumors, and these include Li-Fraumeni syndrome, Neurofibromatosis, Tuberous Sclerosis, von Hippel-Lindau syndrome, and Turcot syndrome, but only $5 \%$ to $10 \%$ of brain tumors are inherited. ${ }^{4}$

\section{Vascular endothelial growth factor (VEGF) pathways and high-grade gliomas}

VEGF is a potent endothelial cell mitogen and key regulator of both physiologic vasculogenesis in the embryonic circulatory system ${ }^{5}$ and pathologic angiogenesis leading to the growth of blood vessels from existing vasculature. ${ }^{6,7}$ VEGF has also been shown to stimulate monocyte/macrophage migration, ${ }^{8-10}$ stimulate tumor cell migration, ${ }^{11-15}$ and enhance vascular permeability in tight-junction endothelial environments such as those of the intact blood-brain barrier. ${ }^{16-18}$

There are five known subtypes of VEGF (VEGF-A, $-\mathrm{B},-\mathrm{C},-\mathrm{D}$ and $-\mathrm{E}$ ) and three known VEGF receptors (VEGFR-1, -2 and -3), all of which are tyrosine kinases. ${ }^{19}$ VEGF A, VEGFR-1 (Flt-1) and VEGFR-2 (KDR/Flk-1) are all highly expressed in the CNS. For CNS tumors, VEGF-A appears to bind to VEGFR-1 and VEGFR-2 and serve a particularly critical role for both angiogenesis and regulation of vascular permeability of the blood - brain barrier. ${ }^{6,16-18}$ VEGF-A stimulates endothelial proliferation via binding to VEGFR-2, and VEGFR-1 is involved in recruitment of macrophages/monocytes that in turn secrete pro-angiogenic factors. VEGF-A is known to stimulate vascular leakage, in particular through VEGFR-1 and VEGFR-2, ${ }^{19}$ and this may contribute to the destabilization (increasing leakiness) of the blood - brain barrier that is often seen in malignant gliomas. Malignant gliomas as well as many other cancers are known to secrete VEGF as a means to stimulate the development of tumor vascular supply (angiogenesis). In turn, expression of VEGF appears to be upregulated by hypoxia as well as a variety of signal and transcription factors. ${ }^{19}$ Specifically upregulated by hypoxia are:

1. HIF- $1 \alpha^{20,21}$

2. STAT $32,23^{22,2}$

3. $\mathrm{Src}^{20,24}$

4. EGFR pathway ${ }^{25,26}$

5. FoxM1B transcription factor ${ }^{27}$

6. Hurl suppresses degradation of VEGF-A mRNA ${ }^{28,29}$

While it is important to identify increased expression of VEGF-A, VEGFR-1 and VEGFR-2 in malignant gliomas, correlation to tumor growth is instrumental as well. Kerber and colleagues studied mice with transplanted glioma cells and mice transplanted with either wild-type bone marrow cells or with VEGFR-1 lacking a tyrosine kinase domain. ${ }^{30}$ They found, using an original glioma cell line and a VEGF-A overexpressing cell line, that a significant reduction in growth of tumor was observed in those mice lacking wild-type VEGFR-1. Studies such as this suggest that VEGF-A and VEGFR-1 may be critical pathways in the growth of malignant gliomas.

\section{Preclinical pharmacology studies}

Bevacizumab is a recombinant, anti-VEGF monoclonal antibody with 6 VEGF-binding residues that binds to VEGF, preventing VEGF from binding to its target, VEGFR-1 and VEGFR-2, on endothelial cells. ${ }^{31}$ Bao and colleagues examined stem cell-like glioma cells (SCLGC) to determine if such cells may be involved in angiogenesis and tumor development. ${ }^{32}$ SCLGC were isolated from human glioblastoma tissue and implanted intracranially into mice. SCLGC, when compared to matched non-SCLGC controls, exhibited higher concentrations of VEGF, and hypoxia seemed to induce VEGF expression. Bevacizumab eliminated angiogenesis and suppressed growth of SCLGC, when compared to matched non-SCLGC controls. The authors concluded that stem-cell like tumors cells could contribute to angiogenesis in certain forms of cancer, such as gliomas.

Preclinical evaluation of antiangiogenic protein expression in four glioma cell lines after treatment with temozolomide found that levels of HIF-1a, ID-1, ID-2 and c-Myc were all reduced. Since these four factors are believed to be involved in angiogenesis and hypoxic metabolism, it was hypothesized that by reducing the levels of these factors, temozolomide may contribute to the reduction of angiogenesis in glioma. The authors also found that, when bevacizumab was added to temozolomide, the survival of 
mice with glioma improved, compared to mice treated with either compound alone..$^{33}$

To test the hypothesis that inhibition of hypoxia inducible factor-1 (HIF-1), when given with antiangiogenic agents, might be more efficacious, bevacizumab was given alone or with topotecan, a topoisomerase inhibitor, with HIF-1 $\alpha$ inhibitor activity. ${ }^{34}$ Using U251-HRE xenografts, the authors showed that bevacizumab reduced microvessel density and increased hypoxia and expression of the HIF-1 dependent gene in the tumor, but it did not induce apoptosis. The addition of topotecan to bevacizumab significantly reduced tumor growth, compared to mice treated with topotecan or bevacizumab alone. Topotecan also reduced expression of HIF-1 and inhibited proliferation while inducing apoptosis. Since the cytotoxic benefit of topotecan did not change with the addition of bevacizumab, the authors concluded that topotecan exerted its effect by HIF-1 inhibition. Furthermore, they hypothesized that bevacizumab, functioning as an antiangiogenesis agent, may represent a potentially beneficial two-drug treatment strategy. ${ }^{34}$

\section{Clinical phase II studies at tumor recurrence}

It is apparent from preclinical studies that VEGF is important for growth of endothelial cells and regulation of tumor angiogenesis; treatment with bevacizumab in vascular tumors like malignant gliomas was anticipated for many years prior to the initiation of formal clinical studies. Nonetheless, the first documented usage of bevacizumab in patients with glioblastoma was in an uncontrolled clinical trial by Stark-Vance in 2005. She presented a series of 29 patients, all with recurrent malignant glioma, treated with bevacizumab in combination with irinotecan. ${ }^{35}$ Stark-Vance used bevacizumab $5 \mathrm{mg} / \mathrm{kg}$ and irinotecan $125 \mathrm{mg} / \mathrm{m}^{2}$ together, intravenously, every 2 weeks, with a 1- to 2-week break between each cycle. There were 3 complete responses (CR), 16 partial responses (PR) and 7 stable diseases (SD); thus, at least $65 \%$ of patients in this case series achieved a response to treatment. Toxicities attributed to bevacizumab included 1 intracranial hemorrhage, 1 bowel perforation, 2 woundhealing abnormalities and 5 cases of epistaxis. ${ }^{35}$

The schedule of most phase 2 studies was based on an intravenous treatment with bevacizumab on a once every 14day schedule. This was established by Genentech based on the plasma clearance half-life of approximately 21 days. While toxicity secondary to bevacizumat is generally mild, nevertheless, since bevacizumab may interfere with wound healing, it is recommended today to wait a minimum of 28 days before or after a major surgical procedure to administer bevacizumab to lower the risk of adverse events such as wound hemorrhage and breakdown. Bevacizumab was approved by the US Food and Drug Administration for recurrent glioblastoma in May 2009. Below we will chronicle some of the studies that led to that approval.

In an early phase II trial, 9 patients with malignant glioma and 23 with glioblastoma were treated with a combination of bevacizumab and irinotecan (Table 1). ${ }^{36}$ Specifically, these patients were treated with bevacizumab $10 \mathrm{mg} / \mathrm{kg}$ IV every 2 weeks and irinotecan $125 \mathrm{mg} / \mathrm{m}^{2}$ for patients on enzymeinducing antiepileptic medication (EIAED) or $340 \mathrm{mg} / \mathrm{m}^{2}$ for patients not on EIAED. Twenty patients (63\%) achieved a radiographic response to treatment. Of the 23 glioblastomas, 14 patients $(61 \%)$ achieved a partial response or better, with a median progression-free survival (PFS) of 20\%. Three patients developed deep venous thromboses or pulmonary emboli, and 1 patient had a stroke. There were no cases of intracranial hemorrhages. ${ }^{36}$

In another retrospective analysis of bevacizumab combined with cytotoxic chemotherapy (irinotecan, carboplatin, carboplatin with erlotinib, carmustine or temozolomide) for recurrent malignant gliomas, a total of 55 patients were reviewed and $63 \%$ showed a response to treatment and $30 \%$ had stable disease (Table 1). ${ }^{37}$ The results of this trial included a 6-month PFS of $42 \%$ for glioblastoma and $32 \%$ for anaplastic glioma. Twenty-three patients, at progression, continued bevacizumab but changed chemotherapeutic

Table I Compilation of phase II studies using bevacizumab for recurrent glioblastoma

\begin{tabular}{lllll}
\hline Patients & Treatment & $\begin{array}{l}\text { Radiographic response } \\
\text { (MR, PR, CR) }\end{array}$ & $\begin{array}{l}\text { PFS } \\
\text { at 6 months }\end{array}$ & Overall survival \\
\hline 23 & BEV + irinotecan ${ }^{36}$ & $61 \%$ & $30 \%$ & Median 9 mos \\
33 & $\mathrm{BEV}+\mathrm{CT}^{37}$ & $64 \%$ & $42 \%$ & N/A \\
48 & $\mathrm{BEV}^{38}$ & $71 \%$ & $29 \%$ & Median 7 mos \\
85 & $\mathrm{BEV}^{41}$ & $28 \%$ & $43 \%$ & Median 9 mos \\
82 & $\mathrm{BEV}+$ irinotecan & & Median 9 mos \\
\hline
\end{tabular}

Abbreviations: PFS, progression-free survival; $M R$, minor response; PR, partial response; $C R$, complete response; $B E V$, bevacizumab; $C T$, variable cytotoxic chemotherapy. 
agents, and this resulted in no radiographic responses, but 2 patients had prolonged PFS. The authors also noted a pattern of increased volume of infiltrative, nonenhancing tumor in those patients who progressed while on bevacizumab. The conclusion reached was that, while bevacizumab combined with cytotoxic chemotherapy is active in patients with malignant gliomas, changing chemotherapeutic agents at progression proved beneficial in only a small subset of patients and progression of tumor seemed to occur in a nonenhancing, infiltrative pattern. ${ }^{37}$

In another phase II trial of patients with recurrent glioblastoma, 48 patients with recurrent glioblastoma were treated with bevacizumab $10 \mathrm{mg} / \mathrm{kg}$ every 2 weeks until tumor progression, then irinotecan was added to bevacizumab, either $340 \mathrm{mg} / \mathrm{m}^{2}$ or $125 \mathrm{mg} / \mathrm{m}^{2}$, depending on EIAED status (Table 1). ${ }^{38}$ While on bevacizumab alone, 34 patients $(71 \%)$ achieved a radiographic response based on Levin criteria, ${ }^{39}$ compared to 17 patients (35\%) when using Macdonald criteria. ${ }^{40}$ The 6-month PFS was $29 \%$, and 6-month overall survival (OS) was 57\%. 19 patients were treated with bevacizumab and irinotecan at progression, and no radiographic responses were observed.

In a larger multi-institutional phase II trial of bevacizumab alone or in combination with irinotecan for recurrent glioblastoma, 167 patients were randomly assigned to bevacizumab $10 \mathrm{mg} / \mathrm{kg}$ every 2 weeks alone or with irinotecan $340 \mathrm{mg} / \mathrm{m}^{2}$ or $125 \mathrm{mg} / \mathrm{m}^{2}$, depending on EIAED status (Table 1). ${ }^{41}$ For those patients on bevacizumab alone, 6-month PFS was 43\%, objective response rates were $28 \%$ and median OS was 9 months. For those patients on bevacizumab and irinotecan, 6-month PFS was $50 \%$, objective response rates were $38 \%$, and median OS was 8.7 months. The bevacizumab-alone group experienced grade 3 or higher adverse events, including hypertension ( $8 \%$ ) and seizures (6\%). The bevacizumab and irinotecan group also experienced grade 3 or higher adverse events, including seizures (14\%), neutropenia (9\%) and fatigue (9\%). Two patients in the bevacizumab-alone group had a grade 1 intracranial hemorrhage $(2 \%)$, compared to 3 patients $(4 \%)$ in the bevacizumab and irinotecan-group (grade 1, 2 and 4). ${ }^{41}$

It is difficult to make a straightforward comparison of bevacizumab to cytotoxic agents used to treat GBM because of the unusual action of bevacizumab and the fact that it does not directly damage DNA of dividing tumor cells. In addition, bevacizumab reduces cerebral edema through a direct effect on brain capillary endothelial cells thus leading to a high "response rate". On the other hand, alkylating agents only secondarily reduce peritumoral edema when they reduce tumor and neoplastic endothelial cell burden. Furthermore, for PFS, comparing bevacizumab and cytotoxic agents can also be misleading, especially since they are not mutually exclusive therapies but rather may be complementary. Lastly, much of the early single-agent cytotoxic chemotherapy literature occurred before the current magnetic resonance imaging (MRI) era became widespread. Nonetheless, for interest's sake we will cite some conclusions from the cytotoxic literature for recurrent GBM.

For this purpose, we selected BCNU, ${ }^{42-44}$ procarbazine, ${ }^{45,46}$ carboplatin, ${ }^{47}$ and temozolomide ${ }^{46}$ data that were analyzed for a prior review. ${ }^{48}$ Since response to these therapies is under $50 \%$ the median will generally be about 8 weeks for all studies. Therefore, in order to better understand and compare alkylating agent therapy to bevacizumab, we elected to combine response and stable disease patients in order to look at duration of therapy benefit. As summarized in table 2, those studies show combined response (PR, CR) and stable disease (minor response [MR], SD) rates of $27 \%$ to $46 \%$ with median time to progression (MTP) of 22 to 30 weeks. ${ }^{48}$ Thus, even though the metrics used in Tables 1 and 2 are different (PFS at 6 months vs. MTP) and the response criteria differ between the study groups $(\mathrm{MR}+\mathrm{PR}+\mathrm{CR}$ vs. $\mathrm{SD}+\mathrm{MR}+\mathrm{PR}+\mathrm{CR})$ summarized in the two tables, one can appreciate that bevacizumab therapy benefits more patients than the cytotoxic drugs, and the durability of a benefit appears to be somewhat longer. That bevacizumab can be combined with some cytotoxic drugs without increasing myelotoxicity should be viewed as encouraging.

\section{Clinical phase II studies and translational research}

A retrospective review of 44 patients treated with bevacizumab for recurrent glioblastoma were compared to 79 patients who were not treated with bevacizumab. ${ }^{49}$ The authors found a significant improvement in PFS and OS in the group treated with bevacizumab. In addition, patients age 55 years or older and those with a Karnofsky Performance

Table 2 Compilation of selected phase II studies of cytotoxic drugs for treatment of recurrent glioblastoma

\begin{tabular}{lll}
\hline Treatment & $\begin{array}{l}\text { Radiographic response } \\
\text { (SD, MR, PR, CR) }\end{array}$ & MTP $^{\mathbf{a}}$ \\
\hline BCNU $^{42-44}$ & 29 & 22 \\
Procarbazine $^{45,46}$ & $27-33$ & 30 \\
Carboplatin $^{47}$ & 40 & 20 \\
Temozolomide $^{46}$ & 46 & 20 \\
\hline
\end{tabular}

aMTP, median time to tumor progression for the SD and responding patients. 
Status of 80 or less had an improved PFS when treated with bevacizumab. VEGF expression in glioblastoma specimens collected on all patients, analyzed with DNA microarray analysis, was higher in patients at least 55 years of age. Lastly, those patients treated with bevacizumab required a lower dose of dexamethasone, and retained their level of function longer when treated with bevacizumab. ${ }^{49}$

Sathornsumetee and colleagues conducted a phase II trial, searching for biomarkers that could predict outcome and response to treatment in patients with recurrent malignant astrocytomas. ${ }^{50}$ Tumor tissue was collected from 27 patients with GBM and 18 with anaplastic astrocytoma at initial diagnosis. The tissue was studied using immunohistochemistry to semi-quantitate expression of VEGF, VEGF receptor 2, CD31, hypoxia-inducible carbonic anhydrase 9 (CA9), and HIF-2 $\alpha$. A total of $58 \%$ experienced PR or more. Elevated expression of VEGF was associated with a greater likelihood of response to treatment, but not a survival benefit. Elevated CA9 expression was associated with a poor outcome, and thus hypoxia, not angiogenesis, ultimately determined survival in this patient population. Median survival for patients with elevated CA9 expression was 37 weeks, while those with low CA9 expression was 74 weeks. The best prognosis was associated with patients whose tumor tissue was negative for $\mathrm{CA} 9$ and $\mathrm{HIF}-2 \alpha$, whereas those who expressed both CA9 and HIF- $2 \alpha$ had the worst prognosis. There were no significant differences in survival for angiogenic markers VEGF, VEGFR-2 or CD31. ${ }^{50}$

Lucio-Eterovic and colleagues compared U87 glioblastoma cell lines and NSC23 glioma stem cell lines with respect to the effects of bevacizumab on in vitro and in vivo invasion, and sought to identify potential mechanisms of resistance to treatment. ${ }^{14}$ These authors were able to show that both cell lines treated with bevacizumab were able to upregulate expression of molecules important for angiogenesis, such as fibroblastic growth factors, interleukins and angiogenins, thereby bypassing the antiangiogenic effect of bevacizumab. Furthermore, there were increased levels of invasion-related proteins (MMP-2, MMP-9, MMP-12, SPARC and TIMPs), suggesting both cell lines treated with bevacizumab may use this as a mechanism for increasing tumor invasiveness. ${ }^{14}$

\section{Clinical phase II studies with irradiation}

A phase II pilot study of bevacizumab in combination with temozolomide and radiation therapy was reported for patients with newly diagnosed glioblastoma multiforme. ${ }^{51}$ In this study, all patients were treated with standard external beam irradiation (60 Gy in 30 fractions, 3 to 5 weeks followed surgery), concurrent temozolomide $\left(75 \mathrm{mg} / \mathrm{m}^{2}\right.$ for 42 days during radiation therapy), and bevacizumab $10 \mathrm{mg} / \mathrm{kg}$ every 2 weeks, starting on day 1 of radiation therapy (RT). After RT was completed, adjuvant temozolomide chemotherapy was continued at 150 to $200 \mathrm{mg} / \mathrm{m}^{2}$, days 1 to 5 every 28 days, and bevacizumab was continued at $10 \mathrm{mg} / \mathrm{kg}$ every 2 weeks. Though preliminary data analysis was encouraging, the routine use of this regimen was not advocated pending completion of a larger, ongoing phase II trial. ${ }^{51}$

Gutin and colleagues studied the safety and efficacy of bevacizumab in patients with recurrent malignant gliomas who also received stereotactic radiation therapy. ${ }^{52} 25$ patients with recurrent malignant glioma, who already received standard radiation therapy, were treated with bevacizumab $10 \mathrm{mg} / \mathrm{kg}$ every 2 weeks until tumor recurrence. These patients were also treated with $30 \mathrm{~Gy}$ of stereotactic radiation therapy in 5 fractions after the first course of bevacizumab. Three patients discontinued bevacizumab due to grade 3 intratumoral hemorrhage, wound dehiscence, and/or bowel perforation. No radiation necrosis was seen in any patients. In patients with GBM, the response rate was $50 \%$, with a 6-month PFS of $65 \%$, and a median OS of 12.5 months. ${ }^{52}$

\section{Clinical trials with anticoagulation}

A retrospective review evaluated the safety of using anticoagulation in glioma patients who were also treated with bevacizumab. ${ }^{53}$ In this report, 21 patients were treated with anticoagulation and bevacizumab for a median of 72 days. No large lobar hemorrhages were noted, although 14\% (3/21) patients had small areas of hemorrhage and only $5 \%(1 / 21)$ developed symptoms from the small hemorrhage. No patients sustained permanent neurological impairments. Interestingly, 7 patients were also identified who developed symptomatic hemorrhages while on bevacizumab but were not receiving anticoagulation. The authors concluded that anticoagulation is not a contraindication to starting bevacizumab. ${ }^{53}$

\section{Bevacizumab and neuroimaging}

A retrospective analysis of patterns of relapse and prognosis, once tumor progression has occurred, was reported for 37 patients with recurrent GBM on bevacizumab. ${ }^{54}$ The median OS after tumor progression on bevacizumab was 4.5 months, with a pattern of progression characterized by an increase in enhancement at the original site of tumor (46\%), a new enhancing lesion distant to the original tumor location (16\%), and growth of nonenhancing tumor (35\%). Additional chemotherapy was given to those with tumor progression on bevacizumab, and the median PFS for these patients was 
2 months, with a median OS of 5.2 months and a 6-month PFS of $0 \%$. The authors concluded that contrast MRI is not sufficient to fully assess treatment response of bevacizumab for recurrent GBM patients, especially since nonenhancing (T2 FLAIR) growth of tumor can be associated with a worse prognosis; additional chemotherapy following failure of bevacizumab provided only transient tumor control. ${ }^{54}$

In another retrospective analysis, 27 patients with recurrent high-grade glioma were treated with irinotecan and bevacizumab and evaluated for safety and efficacy. ${ }^{55}$ In this report, patients were treated with bevacizumab at $10 \mathrm{mg} / \mathrm{kg}$ every 2 weeks and irinotecan every 2 weeks $\left(125 \mathrm{mg} / \mathrm{m}^{2}\right.$ for those not on EIAEDs, $340 \mathrm{mg} / \mathrm{m}^{2}$ for those on EIAEDs). Six-month PFS was 46\%, and median OS 13 months. Median number of prior therapies was 2 in this patient population. Interestingly, 12 patients had radiographic evidence for intracranial hemorrhage prior to receiving bevacizumab, yet only 1 patient required discontinuation of bevacizumab due to progression of hemorrhage. It was concluded that stable intracranial hemorrhage is probably not a contraindication to treatment with bevacizumab and irinotecan. ${ }^{55}$

Another retrospective analysis of 51 patients with recurrent high-grade gliomas treated with bevacizumab and irinotecan was reported. ${ }^{56}$ In this series, patients were treated with bevacizumab $10 \mathrm{mg} / \mathrm{kg}$ and irinotecan $\left(125 \mathrm{mg} / \mathrm{m}^{2}\right.$ for those not on EIAEDs, $340 \mathrm{mg} / \mathrm{m}^{2}$ for those on EIAEDs) IV every 2 weeks. The 6-month PFS for anaplastic glioma was $79 \%$ and $64 \%$ for glioblastoma. Of the 38 patients who experienced progression of disease, 23 showed distant progression, and 7 showed progression only on T2 FLAIR sequences. Twelve percent discontinued bevacizumab and irinotecan due to adverse events, including one with renal failure and another with gastric perforation. No intracranial hemorrhages were reported. The authors concluded that the high rate of distant progression may indicate an ability of the tumor to adapt to bevacizumab with a mechanism of infiltration. ${ }^{56}$

In a retrospective review of MRI from patients with GBM who reviewed bevacizumab-containing regimens, and evaluated for the time course for imaging changes, 15 patients were identified who responded to a bevacizumab regimen and were available for MRI follow up for at least 7 months. The median time to best tumor response was 158 days (range, 16 to 261 ), and the median best response was a $72 \%$ reduction in tumor volume and vasogenic edema. ${ }^{57}$

\section{Bevacizumab and radiation necrosis}

Bevacizumab appears to be active not only in the treatment of patients with glioblastomas, but for those with treatment-related changes from radiation therapy. The first paper to comment on this association was published in $2007 .{ }^{58}$ Eight patients with malignant brain tumors (4 glioblastomas, 3 anaplastic gliomas and 1 hemangiopericytoma) were classified as having radiation necrosis using MRI criteria, ${ }^{59}$ although radiation necrosis was confirmed in 2 patients by biopsy. Following treatment with bevacizumab, alone or in combination with other chemotherapy agents, there was an average reduction of MRI enhancement of $48 \%$ and FLAIR size of $60 \%$. Furthermore, there was a reduction in the average dexamethasone dose of $8 \mathrm{mg}$.

Another retrospective review of 6 patients ( 3 glioblastoma, 1 anaplastic astrocytoma, 1 anaplastic ependymoma and 1 astrocytoma), all with biopsy-proven radiation necrosis, were treated with bevacizumab. The average reduction in enhancement seen on MRI scans was $79 \%$, and FLAIR images on MRI had an average signal reduction of $49 \%$. The radiographic response rate reached $100 \%$, and a response was maintained for a mean of 5.9 months (6 weeks to 18 months). The average number of bevacizumab infusions given was 6.8 , and all patients were able to taper off dexamethasone. ${ }^{60}$

One of the authors (VAL) has completed a randomized, double-blind, placebo-controlled trial of bevacizumab in nonGBM patients with radiation necrosis. ${ }^{61}$ This study strongly confirms the retrospective studies of bevacizumab effectiveness in treating radiation necrosis of the CNS. It appears clear that bevacizumab can reduce capillary leakage, in a VEGF-dependent process, and thereby effectively manage vasogenic edema in patients with malignant brain tumors and radiation necrosis. ${ }^{30}$ In addition, reduction of VEGF by bevacizumab appears to stop the progression of radiation necrosis in many cases. Cliniclas must be cognizant of the possibility that this may confound our ability to truly evaluate response when using neuroimaging criteria in patients with glioblastoma who have MRI scans that could represent tumor progression and/or radiation necrosis.

\section{Concluding remarks}

Bevacizumab has thus far been shown to be active in patients with glioblastoma, with acceptable toxicity. Most serious adverse events, defined as grade 3 or 4 based on Common Terminology Criteria for Adverse Events (CTCAE) v3.0, are $5 \%$ or less, with exception to hypertension (range $6 \%$ to $16 \%){ }^{62}$ Bevacizumab has been shown to improve survival, both PFS and OS, compared to historical controls in glioblastoma patients, with the most impressive response rates thus far for any such therapy. There are data to support activity of bevacizumab alone in patients with recurrent glioblastoma. 
The conundrum facing clinicians who use or would like to use bevacizumab to treat glioblastoma and anaplastic gliomas is how to best utilize its unique pharmacological actions on brain capillary permeability and its ability to interfere with tumor vessel formation. It is naïve to expect that bevacizumab combination with alkylating agents will achieve substantially more in terms of durable response since there is no unique interaction to be exploited. To date, most trials appear to be combinations of bevacizumab with DNAdamaging agents and/or irradiation. Emerging data to show benefit with bevacizumab used at diagnosis along with temozolomide have been judged encouraging based primarily on acceptable toxicity profiles when combining bevacizumab with standard conventional external beam irradiation or hypofractionated radiation therapy at recurrence. There are presently, however, a lack of randomized-controlled trials to provide definitive answers on the true impact of bevacizumab-containing regimens for patients with glioblastoma. Trials such as RTOG 0825, which is presently open and evaluating newly diagnosed glioblastoma patients treated with standard external beam irradiation plus temozolomide versus standard external beam irradiation, temozolomide and bevacizumab, will address some of these concerns, although it may also point out the propensity of bevacizumab to alter glioma tumors to a more invasive phenotype. ${ }^{54}$

It is hoped that the continued identification of biomarkers and genetic patterns will identify patients who may benefit from anti-angiogenic agents such as bevacizumab, and these studies may also suggest other treatable cellular targets that may be critical to the advancement of treatment for glioblastoma patients. Lastly, given important issues of cost and toxicity, future randomized-controlled trials identifying optimal dose and length of treatment would be very helpful in optimizing use of bevacizumab for glioblastoma patients.

\section{Disclosures}

The authors declare no conflicts of interest.

\section{References}

1. Hegi ME, Diserens AC, Gorlia T, et al. MGMT gene silencing and benefit from temozolomide in glioblastoma. $N$ Engl J Med. 2005;352(10): 997-1003.

2. CBTRUS. Statistical Report: Primary Brain Tumors in the United States, 2000-2004 Central Brain Tumor Registry of the United States; 2008.

3. Bondy ML, Scheurer ME, Malmer B, et al. Brain tumor epidemiology: consensus from the Brain Tumor Epidemiology Consortium. Cancer. 2008;113(7 Suppl):1953-1968.

4. Narod SA, Stiller C, Lenoir GM. An estimate of the heritable fraction of childhood cancer. Br J Cancer. 1991;63(6):993-999.

5. Shibuya M. Vascular endothelial growth factor-dependent and -independent regulation of angiogenesis. BMB Rep. 2008;41(4):278-286.
6. Dvorak HF, Sioussat TM, Brown LF, et al. Distribution of vascular permeability factor (vascular endothelial growth factor) in tumors: concentration in tumor blood vessels. J Exp Med. 1991;174(5):1275-1278.

7. Shweiki D, Itin A, Soffer D, Keshet E. Vascular endothelial growth factor induced by hypoxia may mediate hypoxia-initiated angiogenesis. Nature. 1992;359(6398):843-845.

8. Ren Y, Tsui HT, Poon RT, et al. Macrophage migration inhibitory factor: roles in regulating tumor cell migration and expression of angiogenic factors in hepatocellular carcinoma. Int J Cancer. 2003;107(1): 22-29.

9. Proescholdt MA, Heiss JD, Walbridge S, et al. Vascular endothelial growth factor (VEGF) modulates vascular permeability and inflammation in rat brain. J Neuropathol Exp Neurol. 1999;58(6):613-627.

10. Marchio S, Primo L, Pagano M, et al. Vascular endothelial growth factor-C stimulates the migration and proliferation of Kaposi's sarcoma cells. J Biol Chem.1999;274(39):27617-27622.

11. Nagashima G, Suzuki R, Hokaku H, et al. Graphic analysis of microscopic tumor cell infiltration, proliferative potential, and vascular endothelial growth factor expression in an autopsy brain with glioblastoma. Surg Neurol. 1999;51(3):292-299.

12. Sandstrom M, Johansson M, Sandstrom J, Bergenheim AT, Henriksson R. Expression of the proteolytic factors, tPA and uPA, PAI-1 and VEGF during malignant glioma progression. Int $J$ Dev Neurosci. 1999;17(5-6):473-481.

13. Norden AD, Drappatz J, Wen PY. Antiangiogenic therapies for highgrade glioma. Nat Rev Neurol. 2009;5(11):610-620.

14. Lucio-Eterovic AK, Piao Y, de Groot JF. Mediators of glioblastoma resistance and invasion during antivascular endothelial growth factor therapy. Clin Cancer Res. 2009;15(14):4589-4599.

15. Verhoeff JJ, van Tellingen $O$, Claes A, et al. Concerns about antiangiogenic treatment in patients with glioblastoma multiforme. $B M C$ Cancer. 2009;9:444.

16. Criscuolo GR. The genesis of peritumoral vasogenic brain edema and tumor cysts: a hypothetical role for tumor-derived vascular permeability factor. Yale J Biol Med. 1993;66(4):277-314.

17. Risau W. Molecular biology of blood-brain barrier ontogenesis and function. Acta Neurochir Suppl. 1994;60:109-112.

18. Monacci WT, Merrill MJ, Oldfield EH. Expression of vascular permeability factor/vascular endothelial growth factor in normal rat tissues. Am J Physiol. 1993;264(4 Pt 1):C995-1002.

19. Shibuya M. Brain angiogenesis in developmental and pathological processes: therapeutic aspects of vascular endothelial growth factor. Febs J. 2009;276(17):4636-4643.

20. Jiang BH, Agani F, Passaniti A, Semenza GL. v-Src induces expression of hypoxia-inducible factor 1 (Hif-1) and transcription of genes encoding vascular endothelial growth factor and enolase 1- involvement of Hif-1 in tumor progression. Cancer Res. 1997;57(23): $5328-5335$.

21. Gray MJ, Zhang J, Ellis LM, et al. HIF-1alpha, STAT3, CBP/p300 and Ref-1/APE are components of a transcriptional complex that regulates Src-dependent hypoxia-induced expression of VEGF in pancreatic and prostate carcinomas. Oncogene. 2005;24(19):3110-3120.

22. Niu G, Wright KL, Huang M, et al. Constitutive Stat3 activity up-regulates VEGF expression and tumor angiogenesis. Oncogene. 2002;21(13):2000-2008.

23. Lo HW, Cao X, Zhu H, Ali-Osman F. Constitutively activated STAT3 frequently coexpresses with epidermal growth factor receptor in highgrade gliomas and targeting STAT3 sensitizes them to Iressa and alkylators. Clin Cancer Res. 2008;14(19):6042-6054.

24. Mukhopadhyay D, Tsiokas L, Zhou XM, Foster D, Brugge JS, Sukhatme VP. Hypoxic induction of human vascular endothelial growth factor expression through c-Src activation. Nature. 1995;375(6532): $577-581$.

25. Clarke K, Smith K, Gullick WJ, Harris AL. Mutant epidermal growth factor receptor enhances induction of vascular endothelial growth factor by hypoxia and insulin-like growth factor-1 via a PI3 kinase dependent pathway. Br J Cancer. 2001;84(10):1322-1329. 
26. Feldkamp MM, Lau N, Rak J, Kerbel RS, Guha A. Normoxic and hypoxic regulation of vascular endothelial growth Factor (VEGF) by astrocytoma cells is mediated by Ras. Int J Cancer. 1999;81(1): $118-124$.

27. Zhang Y, Zhang N, Dai B, et al. FoxM1B transcriptionally regulates vascular endothelial growth factor expression and promotes the angiogenesis and growth of glioma cells. Cancer Res. 2008;68(21):8733-8742.

28. Nabors LB, Gillespie GY, Harkins L, King PH. HuR, a RNA stability factor, is expressed in malignant brain tumors and binds to adenine- and uridine-rich elements within the 3' untranslated regions of cytokine and angiogenic factor mRNAs. Cancer Res. 2001;61(5):2154-2161.

29. Ido K, Nakagawa T, Sakuma T, Takeuchi H, Sato K, Kubota T. Expression of vascular endothelial growth factor-A and mRNA stability factor HuR in human astrocytic tumors. Neuropathology. 2008;28(6): 604-611.

30. Kerber M, Reiss Y, Wickersheim A, et al. Flt-1 signaling in macrophages promotes glioma growth in vivo. Cancer Res. 2008;68(18): 7342-7351.

31. McCormack PL, Keam SJ. Bevacizumab: a review of its use in metastatic colorectal cancer. Drugs. 2008;68(4):487-506.

32. Bao S, Wu Q, Sathornsumetee S, et al. Stem cell-like glioma cells promote tumor angiogenesis through vascular endothelial growth factor. Cancer Res. 2006;66(16):7843-7848.

33. Mathieu V, De Neve N, Le Mercier M, et al. Combining bevacizumab with temozolomide increases the antitumor efficacy of temozolomide in a human glioblastoma orthotopic xenograft model. Neoplasia. 2008;10(12):1383-1392.

34. Rapisarda A, Hollingshead M, Uranchimeg B, et al. Increased antitumor activity of bevacizumab in combination with hypoxia inducible factor-1 inhibition. Mol Cancer Ther. 2009;8(7):1867-1877.

35. Stark-Vance V. Bevacizumab and CPT-11 in the treatment of relapsed malignant glioma. Neuro Oncol. 2005;7:369.

36. Vredenburgh JJ, Desjardins A, Herndon JE 2nd, et al. Phase II trial of bevacizumab and irinotecan in recurrent malignant glioma. Clin Cancer Res. 2007;13(4):1253-1259.

37. Norden AD, Young GS, Setayesh K, et al. Bevacizumab for recurrent malignant gliomas: efficacy, toxicity, and patterns of recurrence. Neurology. 2008;70(10):779-787.

38. Kreisl TN, Kim L, Moore K, et al. Phase II trial of single-agent bevacizumab followed by bevacizumab plus irinotecan at tumor progression in recurrent glioblastoma. J Clin Oncol. 2009;27(5):740-745.

39. Levin VA, Crafts DC, Norman DM, Hoffer PB, Spire JP, Wilson CB. Criteria for evaluating patients undergoing chemotherapy for malignant brain tumors. J Neurosurg. 1977;47(3):329-335.

40. Macdonald DR, Cascino TL, Schold SC Jr, Cairncross JG. Response criteria for phase II studies of supratentorial malignant glioma. J Clin Oncol. 1990;8(7):1277-1280.

41. Friedman HS, Prados MD, Wen PY, et al. Bevacizumab alone and in combination with irinotecan in recurrent glioblastoma. J Clin Oncol. 2009;27(28):4733-4740.

42. Levin VA. Chemotherapy of primary brain tumors. Neurol Clin. 1985;3(4):855-866.

43. Wilson CB, Gutin P, Boldrey EB, Drafts D, Levin VA, Enot KJ. Singleagent chemotherapy of brain tumors. A five-year review. Arch Neurol. 1976;33(11):739-744.

44. Levin VA, Wilson CB. Nitrosourea chemotherapy for primary malignant gliomas. Cancer Treat Rep. 1976;60(6):719-724.

Cancer Management and Research

\section{Publish your work in this journal}

Cancer Management and Research is an international, peer-reviewed open access journal focusing on cancer research and the optimal use of preventative and integrated treatment interventions to achieve improved outcomes, enhanced survival and quality of life for the cancer patient. The journal welcomes original research, clinical \& epidemiological
45. Rodriguez LA, Prados M, Silver P, Levin VA. Reevaluation of procarbazine for the treatment of recurrent malignant central nervous system tumors. Cancer. 1989;64(12):2420-2423.

46. Yung WK, Albright RE, Olson J, et al. A phase II study of temozolomide vs. procarbazine in patients with glioblastoma multiforme at first relapse. Br J Cancer. 2000;83(5):588-593.

47. Yung WK, Mechtler L, Gleason MJ. Intravenous carboplatin for recurrent malignant gliomas: a phase II study. J Clin Oncol. 1991;9(5):860.

48. Berger MS, Leibel SA, Bruner JM, Finlay JL, Levin VA. Primary Cerebral Tumors. In: Levin VA, ed. Cancer in the Nervous System. Second ed. New York: Oxford University Press; 2002:75-148.

49. Nghiemphu PL, Liu W, Lee Y, et al. Bevacizumab and chemotherapy for recurrent glioblastoma: a single-institution experience. Neurology. 2009;72(14):1217-1222.

50. Sathornsumetee S, Cao Y, Marcello JE, et al. Tumor angiogenic and hypoxic profiles predict radiographic response and survival in malignant astrocytoma patients treated with bevacizumab and irinotecan. $J$ Clin Oncol. 2008;26(2):271-278.

51. Lai A, Filka E, McGibbon B, et al. Phase II pilot study of bevacizumab in combination with temozolomide and regional radiation therapy for up-front treatment of patients with newly diagnosed glioblastoma multiforme: interim analysis of safety and tolerability. Int $J$ Radiat Oncol Biol Phys. 2008;71(5):1372-1380.

52. Gutin PH, Iwamoto FM, Beal K, et al. Safety and efficacy of bevacizumab with hypofractionated stereotactic irradiation for recurrent malignant gliomas. Int J Radiat Oncol Biol Phys. 2009;75(1):156-163.

53. Nghiemphu PL, Green RM, Pope WB, Lai A, Cloughesy TF. Safety of anticoagulation use and bevacizumab in patients with glioma. Neuro Oncol. 2008;10(3):355-360.

54. Iwamoto FM, Abrey LE, Beal K, et al. Patterns of relapse and prognosis after bevacizumab failure in recurrent glioblastoma. Neurology. 2009;73(15):1200-1206.

55. Kang TY, Jin T, Elinzano H, Peereboom D. Irinotecan and bevacizumab in progressive primary brain tumors, an evaluation of efficacy and safety. J Neurooncol. 2008;89(1):113-118.

56. Zuniga RM, Torcuator R, Jain R, et al. Efficacy, safety and patterns of response and recurrence in patients with recurrent high-grade gliomas treated with bevacizumab plus irinotecan. J Neurooncol. 2009;91(3):329-336.

57. Ananthnarayan S, Bahng J, Roring J, et al. Time course of imaging changes of GBM during extended bevacizumab treatment. J Neurooncol. 2008;88(3):339-347.

58. Gonzalez J, Kumar AJ, Conrad CA, Levin VA. Effect of bevacizumab on radiation necrosis of the brain. Int J Radiat Oncol Biol Phys. 2007;67(2):323-326

59. Kumar AJ, Leeds NE, Fuller GN, et al. Malignant gliomas: MR imaging spectrum of radiation therapy- and chemotherapy-induced necrosis of the brain after treatment. Radiology. 2000;217(2):377-384.

60. Torcuator R, Zuniga R, Mohan YS, et al. Initial experience with bevacizumab treatment for biopsy confirmed cerebral radiation necrosis. J Neurooncol. 2009;94(1):63-68.

61. Levin VA, Bidaut L, Hou P, et al. Randomized double-blind placebocontrolled trial of bevacizumab therapy for radiation necrosis of the CNS. Int J Radiat Oncol Biol Phys. 2010. In press.

62. Higa GM, Abraham J. Biological mechanisms of bevacizumabassociated adverse events. Expert Rev Anticancer Ther. 2009;9(7): 999-1007.

\section{Dovepress}

studies, reviews \& evaluations, guidelines, expert opinion \& commentary, case reports \& extended reports. The manuscript management system is completely online and includes a very quick and fair peerreview system, which is all easy to use. Visit http://www.dovepress.com/ testimonials.php to read real quotes from published authors 